01

\title{
Новый подход к расчету проводимости Капицы между твердыми телами
}

\author{
(С) В.И. Хвесюк, Б. Люฯ, А.А. Баринов \\ Московский государственный технический университет им. Н.Э. Баумана, \\ Москва, Россия \\ 『 E-mail: liubinbmstu@gmail.com
}

Поступило в Редакцию 6 мая 2020 г.

В окончательной редакции 16 июня 2020 г.

Принято к публикации 7 июля 2020 г.

В связи с разработкой различных наносистем (компьютерных электронных цепей, термоэлектрических устройств, квантовых каскадных лазеров и др.) очень остро стоит проблема расчета контактных тепловых сопротивлений Капицы между различными материалами. Предложена усовершенствованная модель акустического несоответствия для расчета проводимостей Капицы. Недостаток существующей на данный момент модели заключается в том, что в ней используется приближение Дебая. Это ведет к ограничению применимости модели областью низких температур. Показано, что учет дисперсии волн обеспечивает хорошее согласие данных теории и эксперимента в значительно более широком диапазоне температур, чем при применении современных моделей.

Ключевые слова: проводимость Капицы, микро- и наноперенос тепла, сопротивление Капицы, фононный перенос.

DOI: 10.21883/PJTF.2020.19.50045.18367

В связи с разработкой таких наноэлектронных технологий, как компьютерные электронные цепи, термоэлектрические устройства, квантовые каскадные лазеры и др., одним из важных направлений в современной теории переноса тепла в наноструктурах является развитие методов расчета так называемых проводимостей Капицы (ПК) [1-3].

Ключевой в расчете ПК является величина коэффициента переноса энергии через интерфейс. Существует две модели для определения коэффициента переноса. Первая - модель акустического несоответствия (acoustic mismatch model, AMM) [4]. Она основана на анализе переноса акустических волн через интерфейс [5]. Преимущество этой модели заключается в физической ясности процессов переноса, а недостаток состоит в том, что она дает хорошие результаты только в узком диапазоне температур, близких к абсолютному нулю. Вторая модель основана на анализе переноса фононов через интерфейс. Это модель диффузного несоответствия (diffuse mismatch model, DMM) [1]. Ее преимуществом считается то, что ее можно использовать в более широком диапазоне температур, чем АMM.

Существует две различные версии АММ. Первая версия АММ основана на использовании методов теории упругости [5]. В этом случае задача сводится к решению системы дифференциальных уравнений для определения амплитуд рассеиваемых и преломленных волн при прохождении их через интерфейс. Это позволяет определить долю энергии, переносимой из первого тела во второе, от общей падающей на интерфейс энергии.
Эта версия впервые была сформулирована в работе [6]. Она обозначается EWM (elastic wave model). Вторая версия для определения доли переносимой энергии использует формулы Рэлея [4]. Она не требует решения системы уравнений. Здесь представлены результаты расчетов с использованием обоих подходов. Недостатком этих моделей является отсутствие учета дисперсионных свойств упругих волн, т.е. зависимостей групповых скоростей волн от частоты (так называемое приближение Дебая). Еще одним недостатком классической теории упругости является то, что в ней отсутствует понятие максимальных частот упругих волн, что, естественно, обнаруживается при анализе колебаний решеток твердых тел.

Основная идея настоящей работы - представить усовершенствованную модель, учитывающую дисперсионные свойства упругих волн и различия в максимальных значениях частот [7]. Данный подход мы называем моделью упругой волны с учетом дисперсии (elastic wave model with dispersion, EWMD). Заметим, что в рамках данного подхода скорости упругих волн $v_{g}(\omega)$ во всем диапазоне частот 0 до $\omega_{\max }$ меньше, чем в приближении Дебая, за исключением $v_{g}(\omega=0)$, где эти скорости совпадают. Таким образом, задачами настоящей работы являются сравнение существующих приближений АММ и анализ их развития.

Перенос тепла через контакт двух твердых тел определяется тепловой контактной проводимостью (так называемой проводимостью Капицы [8]). Эта величина связана с тепловым потоком $\Delta q$ и разностью темпера- 
тур на контактных поверхностях $\left(T_{1}-T_{2}\right)$ следующим образом [1]:

$$
\Delta q=h_{K}\left(T_{1}-T_{2}\right),
$$

где индексы 1 и 2 соответствуют двум телам. Здесь тепловой поток $\Delta q$ определяется разностью потоков $q_{1 \rightarrow 2}$ и $q_{2 \rightarrow 1}$. Для каждой моды фононов эти потоки рассчитываются из следующего выражения:

$$
\begin{aligned}
q_{i \rightarrow j}^{m}= & 2 \pi \int_{0}^{\omega_{\max }} \int_{0}^{\pi / 2} \hbar \omega f_{i}\left(\omega, T_{i}\right) v_{g, i}(\omega) D_{i}(\omega) \\
& \times \alpha_{i \rightarrow j}\left(\theta_{0}, \omega\right) \sin \theta_{0} \cos \theta_{0} d \theta_{0} d \omega .
\end{aligned}
$$

Здесь $\omega$ - частота фонона; $\theta_{0}$ - угол между нормалью к границе раздела и направлением распространения фонона; $\omega_{\max }$ - максимальная частота фонона, одинаковая для всех мод, определенная ниже; $T_{i}$ - температура среды $i ; \hbar-$ постоянная Планка, деленная на $2 \pi$;

$$
D_{i}(\omega)=\frac{\omega^{2}}{2 \pi^{2} v_{g, i}(\omega) v_{p, i}^{2}(\omega)},
$$

где $v_{g, i}, v_{p, i}$ являются фононной групповой и фазовой скоростью среды $i$;

$$
f_{i}(\omega, T)=\frac{1}{e^{\hbar \omega / k_{\mathrm{B}} T_{i}}-1}
$$

- функция распределения фононов Бозе-Эйнштейна; $k_{\mathrm{B}}$ - постоянная Больцмана; $\alpha_{i \rightarrow j}\left(\theta_{0}, \omega\right)-$ коэффициент переноса энергии волны (фонона) через интерфейс между двумя телами. Параметр $\alpha_{i \rightarrow j}$ является ключевым для расчета ПК; в приближениях АММ и DMM, кроме того, обычно принимается, что $\alpha_{i \rightarrow j}$ зависит от угла падения. В рамках рассматриваемого в настоящей работе приближения учитываются зависимости $\alpha_{i \rightarrow j}\left(\theta_{0}, \omega\right)$ от угла падения и частоты.

В модели АММ коэффициенты переноса энергии упругими волнами определяются по формулам [4]:

$$
\alpha_{i \rightarrow j}\left(\theta_{0}\right)=\frac{4 \rho_{1} \rho_{2} v_{1} v_{2} \cos \theta_{0} \cos \theta_{2}}{\left(\rho_{1} v_{1} \cos \theta_{0}+\rho_{2} v_{2} \cos \theta_{2}\right)^{2}},
$$

где $\rho_{1}, \rho_{2}-$ плотности первого и второго материалов, $v_{1}, v_{2}-$ скорости упругих волн (продольных или поперечных), в данном приближении не зависящие от частоты, $\theta_{0}$ - угол падения фононов в среде 1 , а $\theta_{2}-$ угол преломления соответствующих фононов в среде 2 . $\theta_{0}$ и $\theta_{2}$ связаны законом Снелла следующим образом: $v_{1} \sin \theta_{2}=v_{2} \sin \theta_{0}$. Согласно формуле (3), коэффициент $\alpha_{i \rightarrow j}\left(\theta_{0}\right)$ зависит только от угла падения $\theta_{0}$.

В приближении $[5,6]$ исходной является картина рассеяния и преломления падающей волны на интерфейсе. На рис. 1 представлена общая картина рассеяния и проникновения продольной волны [5]. Рассматриваются плоские акустические волны, распространяющиеся в двух полубесконечных твердых телах, свойства которых

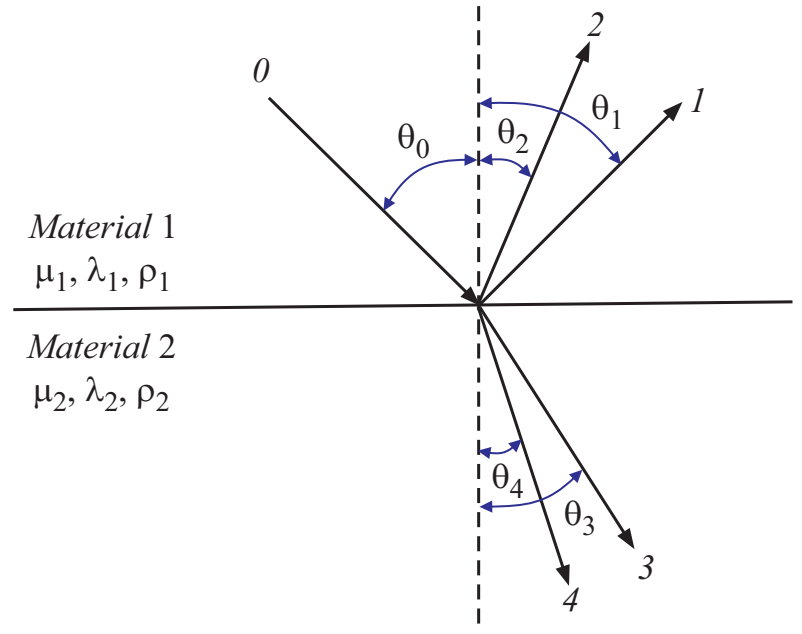

Рис. 1. Схема отражения и преломления волн, падающих на поверхность раздела двух материалов. $O-$ падающая продольная или поперечная волна, 1 - отраженная продольная волна, 2 - отраженная поперечная волна, 3 - преломленная продольная волна, 4 - преломленная поперечная волна.

определяются плотностью $\left(\rho_{1}\right.$ и $\left.\rho_{2}\right)$, а также коэффициентами Ламе $\left(\lambda_{1}, \mu_{1}\right.$, и $\left.\lambda_{2}, \mu_{2}\right)$. Эти тела разделены плоской контактной поверхностью, на которую под углом $\theta_{0}$ к ее нормали падает исходная волна. Поскольку граничные условия двух поперечных волн (ТА) на границе раздела различны, в исследованиях, основанных на теории упругих волн, они обозначаются как SV и $S H$ соответственно. Продольная волна (LA) записывается как $P$-волна [5].

Отношения амплитуды преломленных и отраженных волн $A_{1} / A_{0}, A_{2} / A_{0}, A_{3} / A_{0}, A_{4} / A_{0}$ определяются из решения системы линейных уравнений, следующей из граничных условий непрерывности деформаций и напряжений на контактной поверхности $[5,9]$ :

$$
\begin{gathered}
\left(\begin{array}{cccc}
-\sin \theta_{1} & -\cos \theta_{2} & \sin \theta_{3} & -\cos \theta_{4} \\
\cos \theta_{1} & -\sin \theta_{2} & \cos \theta_{3} & \sin \theta_{4} \\
\sin 2 \theta_{1} & \frac{v_{L}}{v_{T}} \cos 2 \theta_{2} & \frac{\mu_{2} v_{L}}{\mu_{1} v_{L}^{B}} \sin 2 \theta_{3} & -\frac{\mu_{2} v_{L}}{\mu_{1} v_{T}^{B}} \cos 2 \theta_{4} \\
-\frac{v_{L}^{2}}{v_{T}^{2}} \cos 2 \theta_{2} & \frac{v_{L}}{v_{T}} \sin 2 \theta_{2} & \frac{\mu_{2} v_{L} v_{L}^{B}}{\mu_{1}\left(v_{T}^{B}\right)^{2}} \cos 2 \theta_{4} & \frac{\mu_{2} v_{L}}{\mu_{1} v_{T}^{B}} \sin 2 \theta_{4}
\end{array}\right) \\
\times\left(\begin{array}{c}
A_{1} \\
A_{2} \\
A_{3} \\
A_{4}
\end{array}\right)=A_{0}\left(\begin{array}{c}
\sin \theta_{0} \\
\cos \theta_{0} \\
\sin 2 \theta_{0} \\
\frac{v_{L}^{2}}{v_{T}^{2}} \cos 2 \theta_{2}
\end{array}\right) .
\end{gathered}
$$

Здесь $v_{L}, v_{T}$ и $v_{L}^{B}, v_{T}^{B}-$ скорости продольной и поперечной упругих волн среды 1 и среды 2. Углы $\theta_{1}, \theta_{2}, \theta_{3}, \theta_{4}$ с помощью закона Снелла выражаются через $\theta_{0}: v_{0} \sin \theta_{n}=v_{n} \sin \theta_{0}$. Вводя поперечные и продольные групповые скорости и коэффициенты Ламе двух сред в формуле (4), отношение амплитуд генерируемой 


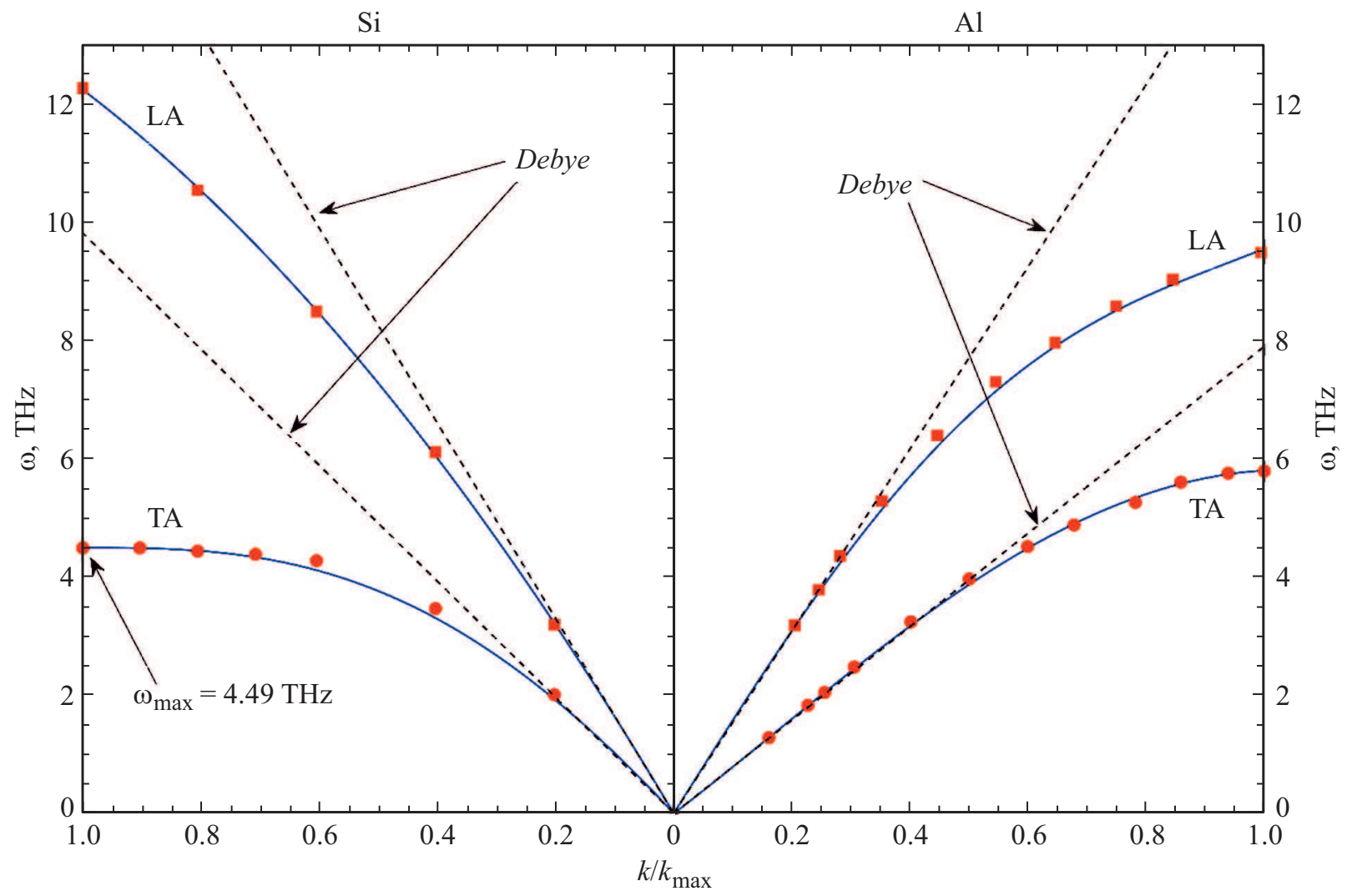

Рис. 2. Дисперсионные соотношения $\mathrm{Si}$ и $\mathrm{Al}$ вдоль направления [100]. Сплошные линии соответствуют продольной (LA) и поперечной (TA) акустическим модам, которые подбираются из реальных дисперсионных соотношений с использованием полиномиальных выражений. Измеренные значения в случае поперечных (кружки) и продольных волн (квадраты) для Si и $\mathrm{Al}$ взяты из работ [12,13] соответственно. Штриховые линии - линейные дисперсионные соотношения с использованием приближения Дебая. Видно, что приближение Дебая дает хорошие результаты только в области малых частот.

акустической волны и падающей волны $\frac{A_{n}}{A_{0}\left(\theta_{0}\right)}$ можно определить численно.

Дисперсия не учитывается в приближении Дебая. В этом приближении учитываются только зависимости амплитуд генерируемых волн от угла $\theta_{0}$. Такое приближение используется в $[6,10,11]$. Рис. 2 показывает, что приближение Дебая и реальные дисперсионные соотношения хорошо согласуются в области частот, близких к нулю. Учет дисперсионных свойств позволяет получить зависимости амплитуд всех волн от частоты и соответственно зависимости от частоты величин $\alpha_{i \rightarrow j}\left(\theta_{0}, \omega\right)$ во всей области частот от 0 до $\omega_{\max }$. Очевидно, что такое решение должно дать более точные результаты.

В нашей модели для аппроксимации дисперсионных соотношений и получения аналитических зависимостей $\omega(k)$ используются полиномы. Далее определяются частотно-зависимые групповые скорости с помощью выражения $v_{g, i}(\omega)=\partial \omega / \partial k$ и фазовые скорости исходя из определения $v_{p, i}(\omega)=\omega / k$. Полученные скорости используются для расчета $D_{i}(\omega)$ в формуле (2). Зависящие от угла и частоты отношения амплитуд генерируемых волн и падающей волны $\frac{A_{n}}{A_{0}}\left(\theta_{0}, \omega\right)$ могут быть определены численно с помощью системы уравнений (4).

Из рис. 2 видно, что каждая волна имеет собственное максимальное значение частоты. На основе теории упругих волн для определения $\alpha_{i \rightarrow j}\left(\theta_{0}, \omega\right)$ используются уравнения энергии. Так, для продольной падающей волны уравнение энергии имеет вид

$$
\begin{aligned}
\left(\frac{A_{1}}{A_{0}}\right)^{2} & +\left(\frac{A_{2}}{A_{0}}\right)^{2} \frac{v_{T} \cos \theta_{2}}{v_{L} \cos \theta_{0}}+\left(\frac{A_{3}}{A_{0}}\right)^{2} \frac{\rho_{2} v_{L}^{B} \cos \theta_{3}}{\rho_{1} v_{L} \cos \theta_{0}} \\
& +\left(\frac{A_{4}}{A_{0}}\right)^{2} \frac{\rho_{2} v_{T}^{B} \cos \theta_{4}}{\rho_{1} v_{L} \cos \theta_{0}}=1,
\end{aligned}
$$

где функции $\theta_{2}, \theta_{3}, \theta_{4}$ выражаются через $\theta_{0}$ с помощью закона Снелла. Очевидно, что в этом случае коэффициент переноса энергии продольной волны $\alpha_{i \rightarrow j}\left(\theta_{0}, \omega\right)$ имеет вид (P-волны)

$$
\begin{aligned}
& \alpha_{i \rightarrow j}^{P}\left(\theta_{0}, \omega\right)=\left(\frac{A_{3}}{A_{0}}\left(\theta_{0}, \omega\right)\right)^{2} \frac{\rho_{2} v_{L}^{B}(\omega) \cos \theta_{3}}{\rho_{1} v_{L}(\omega) \cos \theta_{0}} \\
& +\left(\frac{A_{4}}{A_{0}}\left(\theta_{0}, \omega\right)\right)^{2} \frac{\rho_{2} v_{T}^{B}(\omega) \cos \theta_{4}}{\rho_{1} v_{L}(\omega) \cos \theta_{0}} .
\end{aligned}
$$




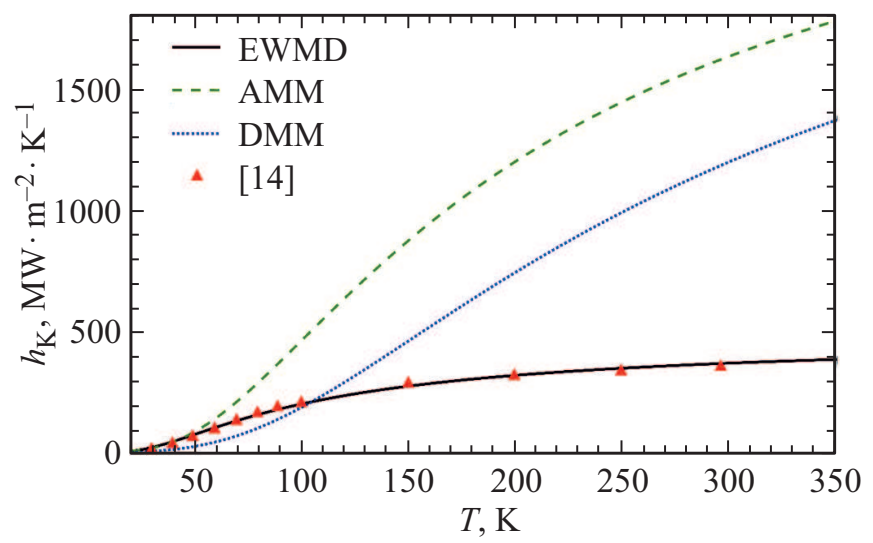

Рис. 3. Прогнозируемые и экспериментальные значения проводимости Капицы на границе раздела $\mathrm{Al}-\mathrm{Si}$ в зависимости от температуры. Точки - измеренные значения из работы [14]. Сплошные линии соответствуют проводимости, полученной с использованием EWMD. Штриховые и пунктирные линии показывают теоретические результаты наиболее широко используемых обычных моделей АMM [4] и DMM [1] соответственно.

Аналогично определяются $\alpha_{i \rightarrow j}^{S V}\left(\theta_{0}, \omega\right), \alpha_{i \rightarrow j}^{S H}\left(\theta_{0}, \omega\right)$, где $S V, S H$ указывают на поперечные волны, у которых вектор колебаний атомов решетки направлен параллельно и перпендикулярно плоскости падения волны.

Существование различных максимальных частот $\omega_{\max }$ для разных поляризаций приводит к важному выводу, от которого зависит конечный результат расчета. Дело в том, что представленная на рис. 1 картина рассеяния и преломления упругих волн - единственно возможная в том смысле, что только при наличии всех четырех отраженных и преломленных волн удовлетворяются граничные условия, связанные с непрерывностью на поверхности контакта деформаций и напряжений.

При задании, например, одной отраженной или одной преломленной волны (при сохранении остальных) эти условия невозможно выполнить. Таким образом, возникающие отраженные и преломленные волны в качестве максимальной имеют одинаковую частоту, равную минимальной из всех максимальных частот. Например, для интерфейса $\mathrm{Al}-\mathrm{Si}$, как показано на рис. $2, \omega_{\max }$ является максимальным значением диапазона частот поперечной волны кремния, $\omega_{\max }=4.49 \mathrm{THz}$. Далее определяются ПК интерфейса $\mathrm{Al}-\mathrm{Si}$ по формуле (2) для всех типов волн и результирующие ПК: $h_{K}^{P}, h_{K}^{S V}, h_{K}^{S H}$. В результате вычисляются ПК $h_{K}=h_{K}^{P}+h_{K}^{S V}+h_{K}^{S H}$.

Результаты расчетов по нашей модели и их сравнение с экспериментальными данными представлены на рис. 3. Видно, что учет дисперсии волн в сочетании с ограничением максимальных значений частот падающей волны, рассеянных и преломленных волн позволяет существенно улучшить согласие теории и эксперимента. Впервые при использовании модели, основанной на рас- смотрении переноса энергии через интерфейс упругими волнами, хорошее согласие с экспериментом получено в широком диапазоне температур от 0 до $300 \mathrm{~K}$. Для пары $\mathrm{Al}-\mathrm{Si}$ максимальное расхождение теории и эксперимента при $300 \mathrm{~K}$ составляет всего $10 \%$. При сравнении использованы экспериментальные данные из работы [14]. Шероховатость в рассматриваемых образцах составляла $0.5 \mathrm{~nm}$. Отметим очень хорошее согласие данных теории АММ и эксперимента в области температур от 0 до $50 \mathrm{~K}$.

В работе для расчета проводимости Капицы между двумя твердыми телами рассматривается улучшенная модель акустического несоответствия, где перенос тепла осуществляется упругими волнами и учитываются дисперсионные соотношения. В этой модели коэффициенты передачи фононов для трех мод акустической волны получаются путем решения системы уравнений теории упругости с частотно-зависимыми граничными условиями, требующими непрерывности компонентов смещения и напряжений. Расчетные значения проводимости Капицы для интерфейса $\mathrm{Al}-\mathrm{Si}$ согласуются с существующими экспериментальными данными в широком интервале температур $(0-300 \mathrm{~K})$. Сравнения с моделями, основанными на модели акустического несоответствия и модели диффузного несоответствия в приближении Дебая, показывают важность полных граничных условий и дисперсии, а также подтверждают целесообразность нашей модели для прогнозирования и описания межфазной теплопередачи.

\section{Конфликт интересов}

Авторы заявляют, что у них нет конфликта интересов.

\section{Список литературы}

[1] Swartz E.T., Pohl R.O. // Rev. Mod. Phys. 1989. V. 61. P. 605668.

[2] Cahill D.G., Ford W.K., Goodson K.E., Mahan G.D., Majumdar A., Maris H.J., Merlin R., Phillpot S.R. // J. Appl. Phys. 2003. V. 93. P. 793-818.

[3] Cahill D.G., Braun P.V., Chen G., Clarke D.R., Fan S., Goodson K.E., Keblinski P., King W.P., Mahan G.D., Majumdar A., Maris H.J., Phillpot S.R., Pop E., Shi L. // Appl. Phys. Rev. 2014. V. 1. P. 011305.

[4] Little W. // Can. J. Phys. 1959. V. 37. P. 334-349.

[5] Achenbach $J$. Wave propagation in elastic solids. Elsevier, 2012. V. 16. 440 p.

[6] Халатников И.М. // ЖЭТФ. 1952. Т. 22. В. 6. С. 687-704.

[7] Chung J.D., McGaughey A.J.H., Kaviany M. // J. Heat Transfer. 2004. V. 126. P. 376-380.

[8] Kapitza P. // J. Phys. USSR. 1941. V. 4. P. 181-210.

[9] Müller G. Theory of elastic waves. Potsdam: Geoforschungszentrum, 2007. 228 p.

[10] Cheeke J., Ettinger H., Hebral B. // Can. J. Phys. 1976. V. 54. P. 1749-1771.

[11] Peterson R.E., Anderson A.C. // J. Low Temp. Phys. 1973. V. 11. P. 639-665. 
[12] Weber W. // Phys. Rev. B. 1977. V. 15. P. 4789-4803.

[13] Gilat G., Nicklow R.M. // Phys. Rev. 1966. V. 143. P. 487-494.

[14] Minnich A.J., Johnson J.A., Schmidt A.J., Esfarjani K., Dresselhaus M.S., Nelson K.A., Chen G. // Phys. Rev. Lett. 2011. V. 107. P. 095901. 